\title{
Coincidence Points for Perturbations of Linear Fredholm Maps of Index Zero
}

By

\author{
Ravi P. Agarwal* and Donal O'Regan**
}

\begin{abstract}
Coincidence points for single and set valued maps are discussed in this paper. We show if $F$ is essential and $F \cong G$ then $G$ has a coincidence point.
\end{abstract}

\section{§1. Introduction}

The notion of an essential map was introduced by Granas in [4]. He showed in [4] that if $F$ is essential and $F \cong G$ then $G$ is essential. Since the property of being essential is quite general Granas was only able to show this homotopy property for particular classes of maps. However from an application point of view he was asking too much. What one needs usually in applications is the following question to be answered: if $F$ is essential and $F \cong G$, does $G$ have a fixed (or more generally a coincidence) point? Recall two maps $F: X \rightarrow 2^{Y}$ and $G: X \rightarrow 2^{Y}$ have a coincidence if $F\left(x_{0}\right) \cap G\left(x_{0}\right) \neq \emptyset$ for some $x_{0} \in X$; the point $x_{0}$ is called a coincidence point. In this paper we discuss this question in detail. In Section 2 we discuss single valued maps which satisfy the Mönch-Precup condition and in Section 3 multivalued $k$-set contractive maps. Our results extend those in Precup [9] and Volkmann [11].

For the remainder of this section we present some concepts which will be needed in Section 2 and in Section 3. Let $(Z, d)$ be a metric space and let $\Omega_{Z}$ be the bounded subsets of $Z$. The Kuratowskii measure of noncompactness is the map $\alpha: \Omega_{Z} \rightarrow[0, \infty]$ defined by (here $B \in \Omega_{Z}$ ),

$$
\alpha(B)=\inf \left\{r>0: B \subseteq \bigcup_{i=1}^{n} B_{i} \text { and } \operatorname{diam}\left(B_{i}\right) \leq r\right\} .
$$

Communicated by T. Kawai, November 16, 1998. Revised March 31, 1999 and April 28, 1999 1991 Mathematics Subject Classification(s): 55H25, 34B15

* Department of Mathematics, National University of Singapore, 10 Kent Ridge Crescent, Singapore 119260.

** Department of Mathematics, National University of Ireland, Galway, Ireland. 
Let $S$ be a nonempty subset of $Z$ and suppose $G: S \rightarrow 2^{Z}$ (here $2^{Z}$ denotes the family of nonempty subsets of $Z$ ). Then (i). $G: S \rightarrow 2^{Z}$ is $k$-set contractive (here $k \geq 0)$ if $\alpha(G(A)) \leq k \alpha(A)$ for all nonempty, bounded sets $A$ of $S$ (here $G(A)=\bigcup_{x \in A} G(x)$ ), and (ii). $G: S \rightarrow 2^{Z}$ is condensing if $G$ is 1 -set contractive and $\alpha(G(A))<\alpha(A)$ for all bounded sets $A$ of $S$ with $\alpha(A) \neq 0$.

Let $X$ and $E$ be Fréchet spaces and $L: \operatorname{dom} L \subseteq X \rightarrow E$ (dom $L$ is a vector subspace of $X$ ) is a linear Fredholm map of index zero i.e. $L$ is a linear (not necessarily continuous) single valued map with $\operatorname{Im} L$ closed and $\operatorname{dim}(k e r$ $L)=\operatorname{codim}(\operatorname{Im} L)<\infty$. Let $X=X_{1} \oplus X_{2}$ and $E=E_{1} \oplus E_{2}$ (topological direct sums) where $X_{1}=\operatorname{ker} L$ and $E_{2}=\operatorname{Im} L$. Let $P: X \rightarrow X_{1}, Q: E \rightarrow E_{1}$ be continuous linear projections and $J: X_{1} \rightarrow E_{1}$ a linear isomorphism (i.e. a linear homeomorphism). Finally $\Phi: X \rightarrow E_{1}$ will be a linear, continuous single valued map with $L+\Phi: \operatorname{dom} L \rightarrow E$ an isomorphism; for convenience we say $\Phi \in H_{L}\left(X, E_{1}\right)$.

\section{§2. Single Valued Maps}

Let $X$ and $E$ be Fréchet spaces, $U$ an open subset of $X, 0 \in U$ and $\Phi \in H_{L}\left(X, E_{1}\right)$ is fixed (here $L$ and $E_{1}$ are as described in Section 1).

Definition 2.1. We let $M_{\partial U}(\bar{U}, E ; L, \Phi)$ denote the set of all continuous maps $F: \bar{U} \rightarrow E$ which satisfy the Mönch-Precup condition (i.e. if $C \subseteq \bar{U}$ is countable, $W \subseteq k e r L$ is compact and $C \subseteq \overline{c o}\left(\{0\} \cup(L+\Phi)^{-1}(F+\Phi)(C)\right)+W$ then $\bar{C}$ is compact) and with $(L-F)(x) \neq 0$ for $x \in \partial U$ ndom $L$; here $\partial U$ is the boundary of $U$ in $X, \bar{U}$ the closure of $U$ in $X$ and $\overline{c o}(A)$ denotes the closed convex hull of $A$.

Remark 2.1. If $(L+\Phi)^{-1} \Phi(\bar{C})$ is a bounded set in $k e r L$ then it is well known [9] (note $\operatorname{dim}(\operatorname{ker} L)<\infty)$ that $(L+\Phi)^{-1} \Phi(\bar{C})$ is relatively compact, so as a result in this case we could define the Mönch-Precup condition in

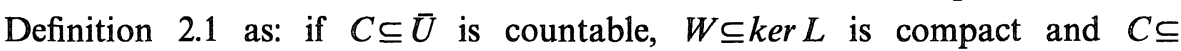
$\overline{c o}\left(\{0\} \cup(L+\Phi)^{-1} F(C)\right)+W$ then $\bar{C}$ is compact. To see why we need only note that $(L+\Phi)^{-1} \Phi(C) \subseteq k e r L$ is relatively compact since $(L+\Phi)^{-1} \Phi(\bar{U})$ is relatively compact.

Definition 2.2. A map $F \in M_{\partial v}(\bar{U}, E ; L, \Phi)$ is essential if for every $G \in M_{\partial U}(\bar{U}, E ; L, \Phi)$ with $\left.G\right|_{\partial U}=\left.F\right|_{\partial U}$ we have that there exists $x \in \bar{U} \cap \operatorname{dom} L$ with $L x=G(x)$.

Theorem 2.1. Let $X$ and $E$ be Fréchet spaces, $U$ an open subset of $X, 0 \in U$ 
and $\Phi \in H_{L}\left(X, E_{1}\right)$. Suppose $F \in M_{\partial v}(\bar{U}, E ; L, \Phi)$ is an essential map and $H: \bar{U} \times$ $[0,1] \rightarrow E$ a continuous map with the following properties:

$$
H(x, 0)=F(x) \text { for } x \in \bar{U}
$$

$$
\left.L x \neq H_{t}(x) \text { for any } x \in \partial U \cap \operatorname{dom} L \text { and } t \in(0,1] \text { (here } H_{t}(x)=H(x, t)\right)
$$

and

$$
\begin{aligned}
& \text { for any continuous } \mu: \bar{U} \rightarrow[0,1] \text { with } \mu(\partial U)=0 \text { the map } \\
& R_{\mu}: \bar{U} \rightarrow E \text { defined by } R_{\mu}(x)=H(x, \mu(x)) \text { satisfies the Mönch-Precup } \\
& \text { condition (i.e. if } C \subseteq \bar{U} \text { is countable, } W \subseteq k e r L \text { is compact and } \\
& \left.C \subseteq \overline{c o}\left(\{0\} \cup(L+\Phi)^{-1}\left(R_{\mu}+\Phi\right)(C)\right)+W \text { then } \bar{C} \text { is compact }\right) \text {. }
\end{aligned}
$$

Then there exists $x \in U$ तdom $L$ with $L x=H_{1}(x)$.

Remark 2.2. If $(L+\Phi)^{-1} \Phi(\bar{U})$ is a bounded set in $X$ we could define the Mönch-Precup condition in (2.3) as: if $C \subseteq \bar{U}$ is countable, $W \subseteq k e r L$ is compact and $C \subseteq \overline{c o}\left(\{0\} \cup(L+\Phi)^{-1} R_{\mu}(C)\right)+W$ then $\bar{C}$ is compact.

Proof. Let

$$
B=\left\{x \in \bar{U} \cap \operatorname{dom} L: L x=H_{t}(x) \text { for some } t \in[0,1]\right\} .
$$

It is immediate that

$$
B=\left\{x \in \bar{U}: x=(L+\Phi)^{-1}\left(H_{t}+\Phi\right)(x) \text { for some } t \in[0,1]\right\} .
$$

When $t=0, H_{0}=F$ and since $F \in M_{\partial U}(\bar{U}, E ; L, \Phi)$ is essential there exists $x \in \bar{U} \cap \operatorname{dom} L$ with $L x=F(x)$. Thus $B \neq \emptyset$. The continuity of $H, \Phi$ and $(L+\Phi)^{-1}$ guarantees that $B$ is closed. In addition (2.2) (together with $\left.F \in M_{\partial U}(\bar{U}, E ; L, \Phi)\right)$ implies $B \cap \partial U=\emptyset$. Thus there exists a continuous $\mu: \bar{U} \rightarrow$ $[0,1]$ with $\mu(\partial U)=0$ and $\mu(B)=1$. Define a map $R: \bar{U} \rightarrow E$ by

$$
R(x)=H(x, \mu(x))
$$

Now $R$ is continuous and satisfies the Mönch-Precup condition (see (2.3)). Moreover for $x \in \partial U \cap \operatorname{dom} L$,

$$
(L-R)(x)=\left(L-H_{0}\right)(x)=(L-F)(x) \neq 0
$$

so $R \in M_{\partial U}(\bar{U}, E ; L, \Phi)$. Also notice $\left.R\right|_{\partial U}=\left.H_{0}\right|_{\partial U}=\left.F\right|_{\partial U}$ and since $F \in M_{\partial U}$ $(\bar{U}, E ; L, \Phi)$ is essential there exists $x \in \bar{U} \cap \operatorname{dom} L$ with $(L-R)(x)=0$ (i.e. $\left(L-H_{\mu(x)}(x)=0\right)$. Thus $x \in B$ and so $\mu(x)=1$. Consequently $\left(L-H_{1}\right)(x)=0$ and we are finished (since (2.2) implies $x \in U \cap \operatorname{dom} L$ ). 
We now use Theorem 2.1 to obtain a nonlinear alternative of LeraySchauder type for Mönch-Precup maps. To prove our result we need the following well known result from the literature [2]. For the remainder of this section $X$ and $E$ will be Banach spaces.

Theorem 2.2. Let $X$ be a Banach space, $D$ a closed, convex set of $X$ with $0 \in D$. Suppose $J_{0}: D \rightarrow D$ is a continuous map which satisfies Mönch's condition (i.e. if $C \subseteq \bar{U}$ is countable and $C \subseteq \overline{c o}\left(\{0\} \cup J_{0}(C)\right)$ then $\bar{C}$ is compact). Then $J_{0}$ has a fixed point in $D$.

Theorem 2.3. Let $X$ and $E$ be Banach spaces, $U$ an open subset of $X$, $0 \in U \cap \operatorname{dom} L$ and $\Phi \in H_{L}\left(X, E_{1}\right)$ is such that $(L+\Phi)^{-1} \Phi(\bar{U})$ is a bounded set in ker L. Suppose $G: \bar{U} \rightarrow E$ is a continuous map which satisfies the Mönch-Precup

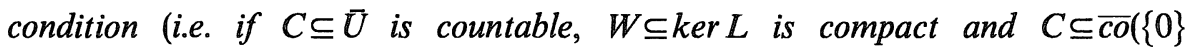
$\left.\cup(L+\Phi)^{-1} G(C)\right)+W$ then $\bar{C}$ is compact) and assume

$$
L x \neq t G(x)+(1-t)(-\Phi(x)) \quad \text { for } x \in \partial U \text { ndom } L \text { and } t \in(0,1)
$$

is satisfied. Then there exists $x \in \bar{U} \cap \operatorname{dom} L$ with $L x=G(x)$.

Proof. We assume $L x \neq G(x)$ for $x \in \partial U \cap \operatorname{dom} L$ (otherwise we are finished). Then

$$
L x \neq t G(x)+(1-t)(-\Phi(x)) \text { for } x \in \partial U \cap \operatorname{dom} L \text { and } t \in[0,1] \text {. }
$$

(Note if $t=0$ and if $L x_{0}=-\Phi\left(x_{0}\right)$ for $x_{0} \in \partial U \cap \operatorname{dom} L$, then $(L+\Phi)\left(x_{0}\right)=0$ so $x_{0}=0$, which is a contradiction since $\left.0 \in U \cap \operatorname{dom} L\right)$. Let $H(x, t)=t G(x)+(1-t)$ $(-\Phi(x))$ for $(x, t) \in \bar{U} \times[0,1]$ and $F(x)=-\Phi(x)$ for $x \in \bar{U}$. Notice (2.1) and (2.2) hold. To see (2.3) let $C \subseteq \bar{U}$ be countable and $W \subseteq k e r L$ compact with

$$
C \subseteq \overline{c o}\left(\{0\} \cup(L+\Phi)^{-1}\left(R_{\mu}+\Phi\right)(C)\right)+W .
$$

Notice for $x \in C,\left(R_{\mu}+\Phi\right)(x)=\mu(x)[G(x)+\Phi(x)]$ and as a result

$$
(L+\Phi)^{-1}\left(R_{\mu}+\Phi\right)(C) \subseteq \operatorname{co}\left((L+\Phi)^{-1}(G+\Phi)(C) \cup\{0\}\right) .
$$

In addition since $\{0\} \cup \operatorname{co}\left((L+\Phi)^{-1}(G+\Phi)(C) \cup\{0\}\right)=\operatorname{co}\left((L+\Phi)^{-1}(G+\Phi)(C)\right.$ $\cup\{0\})$ and $\operatorname{co}\left((L+\Phi)^{-1}(G+\Phi)(C) \cup\{0\}\right)$ is convex we have

$$
\begin{aligned}
C & \subseteq \overline{c o}\left(\{0\} \cup(L+\Phi)^{-1}\left(R_{\mu}+\Phi\right)(C)\right)+W \subseteq \overline{c o}\left(\operatorname{co}\left((L+\Phi)^{-1}(G+\Phi)(C) \cup\{0\}\right)\right)+W \\
& =\overline{c o}\left((L+\Phi)^{-1}(G+\Phi)(C) \cup\{0\}\right)+W .
\end{aligned}
$$

Now since $G$ satisfies the Mönch-Precup condition we have that $\bar{C}$ is 
compact. Thus (2.3) holds. We can apply Theorem 2.1 if we show $F \in M_{\partial U}(\bar{U}, E ; L, \Phi)$ is essential. First notice $F \in M_{\partial U}(\bar{U}, E ; L, \Phi)$ [It is immediate that $F$ satisfies the Mönch-Precup condition in Remark 2.1. Also note if $(L-F)\left(x_{0}\right)=0$ for some $x_{0} \in \partial U \cap \operatorname{dom} L$ then $x_{0}=0$, a contradiction]. To show $F$ is essential let $\theta \in M_{\partial U}(\bar{U}, E ; L, \Phi)$ with $\left.\theta\right|_{\partial U}=\left.F\right|_{\partial U}=-\left.\Phi\right|_{\partial U}$. We must show that there exists $x \in \bar{U} \cap \operatorname{dom} L$ with $L x=\theta(x)$. Let $D=\overline{c o}\left((L+\Phi)^{-1}(\theta+\Phi)(\bar{U})\right)$ and let $J_{0}: D \rightarrow D$ be defined by

$$
J_{0}(x)=\left\{\begin{array}{l}
(L+\Phi)^{-1}(\theta+\Phi)(x), \quad x \in \bar{U} \\
0, \quad x \notin \bar{U} .
\end{array}\right.
$$

Note $0 \in D$ and $J_{0}: D \rightarrow D$ is continuous. We now show $J_{0}$ satisfies Mönch's condition. To see this let $C \subseteq D$ be countable with $C \subseteq \overline{c o}\left(\{0\} \cup J_{0}(C)\right)$. Then

$$
C \subseteq \overline{c o}\left(\{0\} \cup(L+\Phi)^{-1}(\theta+\Phi)(\bar{U} \cap C)\right) .
$$

Note as well that $(L+\Phi)^{-1} \Phi(\bar{U} \cap C) \subseteq k e r L$ is relatively compact and this together with (2.7) gives

$$
C \cap \bar{U}(\subseteq C) \subseteq \overline{c o}\left(\{0\} \cup(L+\Phi)^{-1} \theta(\bar{U} \cap C)\right)+W
$$

where $W \subseteq k e r L$ is a compact set. Since $\theta$ satisfies the Mönch-Precup condition we have $\overline{C \cap \bar{U}}$ compact. Thus since $(L+\Phi)^{-1} \theta$ is continuous, $(L+\Phi)^{-1} \theta(\overline{C \cap \bar{U}})$ is compact and Mazur's Theorem implies $\overline{c o}\left(\{0\} \cup(L+\Phi)^{-1} \theta(\overline{C \cap \bar{U}})+W\right.$ is compact. Now since $C \subseteq \overline{c o}\left(\{0\} \cup(L+\Phi)^{-1} \theta(\bar{U} \cap C)\right)+W$ we have that $\bar{C}$ is compact. Consequently $J_{0}: D \rightarrow D$ is continuous and satisfies Mönch's condition. Theorem 2.2 implies that there exists $x \in D$ with $J_{0}(x)=x$. Now if $x \notin U$, we have $0=J_{0}(x)=x$, which is a contradiction since $0 \in U$. Thus $x \in U$ so $x=J_{0}(x)=(L+\Phi)^{-1}(\theta+\Phi)(x)$ i.e. $x \in U \cap \operatorname{dom} L$ and $L x=\theta(x)$. Hence $F$ is essential and we may apply Theorem 2.1 to deduce the result.

Theorem 2.3 gives us a nice criteria for recognizing essential maps (see Remark 2.4.). Our next result is particularly useful in applications.

Theorem 2.4. Let $X$ and $E$ be Banach spaces. $U$ an open subset of $X$ and $0 \in U \cap \operatorname{dom} L$. Let $P, Q, J$ be as in Section 1 with $\Phi=J P$ and assume $(L+J P)^{-1} J P(\bar{U})$ is a bounded set in ker $L$. Suppose $G: \bar{U} \rightarrow E$ is a continuous map with $(L+J P)^{-1} G: \bar{U} \rightarrow X k$-set contractive (here $\left.0 \leq k<1\right)$ and $(L+J P)^{-1} G(\bar{U})$ a bounded set in $X$. Also assume

$$
Q G \in M_{\partial U}(\bar{U}, E ; L, J P) \text { is an essential map }
$$




$$
Q G(x) \neq 0 \text { for all } x \in \partial U \cap X_{1}
$$

and

$$
L x \neq t G(x) \text { for } x \in \partial U \cap\left(\operatorname{dom} L \backslash X_{1}\right) \text { and } t \in(0,1)
$$

are satisfied. Then there exists $x \in \bar{U} \cap$ dom $L$ with $L x=G(x)$.

Proof. Assume $L x \neq G(x)$ for $x \in \partial U$ ndom $L$. Let $H(x, t)=t G(x)+(1-t)$ $Q G(x)$. To see (2.2) notice if $L x=H_{t}(x)$ for some $x \in \partial U \cap \operatorname{dom} L$ and $t \in(0,1]$ then

$$
L x=t G(x)+(1-t) Q G(x) .
$$

It is easy to see that (2.11) is equivalent to

$$
L x=t(I-Q) G(x) \text { and } Q G(x)=0 .
$$

This together with (2.9) gives $L x=t G(x)$ for $x \in \partial U \cap\left(\operatorname{dom} L \backslash X_{1}\right)$, a contradiction. As a result (2.2) holds. To see (2.3) let $C \subseteq \bar{U}$ be countable and $W \subseteq k e r L$ compact with

$$
C \subseteq \overline{c o}\left(\{0\} \cup(L+\Phi)^{-1}\left(R_{\mu}+\Phi\right)(C)\right)+W
$$

here $\Phi=J P$. Now since

$$
\begin{aligned}
\left(R_{\mu}+\Phi\right)(x) & =\mu(x) G(x)+(1-\mu(x)) Q G(x) \Phi(x) \\
& =\mu(x)[G+\Phi](x)+(1-\mu(x))[Q G+\Phi](x)
\end{aligned}
$$

we have

(2.14) $(L+\Phi)^{-1}\left(R_{\mu}+\Phi\right)(C) \subseteq c o\left((L+\Phi)^{-1}(G+\Phi)(C) \cup(L+\Phi)^{-1}(Q G+\Phi)(C)\right)$.

Now (2.13), (2.14), $(L+\Phi)^{-1} G: \bar{U} \rightarrow X \quad k$-set contractive, $(L+J P)^{-1} Q G=$ $P(L+J P)^{-1} G$ with $P$ having finite dimensional range (so $P$ is completely continuous), immediately guarantees that $\bar{C}$ is compact. Thus (2.3) holds so we may apply Theorem 2.1 to deduce the result.

Remark 2.3. It is also easy to establish, under extra assumptions, the analogue of Theorem 2.4 with general $\Phi$ and $(L+\Phi)^{-1} G$ being $k$-set contractive replaced by the more general assumption that $G$ satisfies the Mönch-Precup condition. We leave the details to the reader.

Remark 2.4. It is reasonably easy to put conditions on $G$ in Theorem 2.4 to guarantee that $(2.8)$ is satisfied. For example if 


$$
\langle Q G(x), J(x)\rangle \leq 0 \text { for } x \in \partial U \cap X_{1}
$$

then (2.8) is satisfied; here $\langle.,$.$\rangle denotes the euclidean inner product on E_{1}$ (note $\left.\operatorname{dim} E_{1}<\infty\right)$. To see this let $\theta \in M_{\partial U}(\bar{U}, E ; L, J P)$ with $\left.\theta\right|_{\partial U}=\left.Q G\right|_{\partial U}$. We will now use Theorem 2.3 to show that there exists $x \in \bar{U} \cap \operatorname{dom} L$ with $L x=\theta(x)$. We need only check (2.4). Suppose there exists $x \in \partial U \cap \operatorname{dom} L$ and $t \in(0,1)$ with

$$
L x=t \theta(x)+(1-t)(-J P(x))=t Q G(x)+(1-t)(-J P(x)) .
$$

Then

$$
(L+J P)(x)=t(Q G+J P)(x)
$$

It is easy to see that

$$
\left.L x=0 \text { (i.e. } x \in X_{1} \text { so } P(x)=x\right) \text { and } t Q G(x)=(1-t) J(x) .
$$

Thus $x \in \partial U \cap X_{1}$ and

$$
t\langle Q G(x), J(x)\rangle=(1-t)|J(x)|^{2}>0 .
$$

This contradicts (2.15), so (2.4) holds. Theorem 2.3 guarantees that there exists $x \in \bar{U} \cap \operatorname{dom} L$ with $L x=\theta(x)$, so (2.8) holds.

As an application of the results above consider the system of $n$ first order differential equations

$$
\left\{\begin{array}{l}
y^{\prime}=f(t, y) \text { for } t \in[0,1] \\
y(0)=y(1)
\end{array}\right.
$$

Let $C_{P}[0,1]=\{u \in C[0,1]: u(0)=u(1)\}, C_{0}=\{u \in C[0,1]: u(0)=0\}$, and let $L: C_{P} \rightarrow$ $C_{0}$ be given by

$$
L u(t)=u(t)-u(0)
$$

For each $a \in \mathbf{R}^{n}$ let $\bar{a}$ denote the constant function in $C_{P}$ with value $a$. It is immediate that

$$
\operatorname{ker} L=\left\{\bar{a}: a \in \mathbf{R}^{n}\right\} \text { and } \operatorname{Im} L=\left\{v \in C_{0}: v(1)=0\right\} .
$$

Since each $v \in C_{0}$ can be expressed as $v(t)=t v(1)+[v(t)-t v(1)]$ we have the direct sum decomposition $C_{0}=\left(t \mathbf{R}^{n}\right) \oplus \operatorname{Im} L$. Thus $L$ is a Fredholm map of index zero and we may set $P u=u(1),(Q v)(t)=t v(1), J \bar{a}=t a$ and $\Phi=J P$.

We will assume $f:[0,1] \times \mathbf{R}^{n} \rightarrow \mathbf{R}^{n}$ is continuous. Let $G: C_{P} \rightarrow C_{0}$ be defined by 


$$
G u(t)=\int_{0}^{t} f(s, u(s)) d s
$$

It is well known that $G: C_{P} \rightarrow C_{0}$ is continuous and completely continuous.

Theorem 2.6. Let $f:[0,1] \times \mathbb{R}^{n} \rightarrow \mathbb{R}^{n}$ be continuous. Suppose there is a constant $R>0$ with $|u|_{0}=\sup _{t \in[0,1]}|u(t)|<R$ for any solution $u \in C^{1}[0,1]$ to

$$
\left\{\begin{array}{l}
y^{\prime}=\lambda f(t, y) \quad \text { for } \quad t \in[0,1] \\
y(0)=y(1)
\end{array}\right.
$$

for $0<\lambda<1$. Also assume

$$
\text { for all } a \in \mathbb{R}^{n} \text { with }|a|=R \text { we have } \int_{0}^{1} f(s, a) d s \neq 0
$$

and

$$
\text { for all } a \in \mathbb{R}^{n} \text { with }|a|=R \text { we have }\left\langle a, \int_{0}^{1} f(s, a) d s\right\rangle \leq 0
$$

hold. Then there exists a solution $u \in C^{1}[0,1]$ to $(2.16)$ with $|u|_{0} \leq R$.

Proof. Let

$$
U=\left\{u \in C_{P}:|u|_{0}<R\right\}, X=C_{P}, E=C_{0}
$$

and $G: C_{P} \rightarrow C_{0}$ be as in (2.17). Notice (see the definition of ker $L$ ) we have $\partial U \cap k e r L=\{\bar{a}: \bar{a} \in \partial U\}$. If (2.9) fails then $Q G(\bar{a})=0$ for some $\bar{a} \in \partial U \cap X_{1}$ i.e. $|\bar{a}|_{0}=R$ and

$$
Q G(\bar{a})=t \int_{0}^{1} f(s, a) d s=0 \text { for all } t \in[0,1] .
$$

Consequently $\int_{0}^{1} f(s, a) d s=0$ for some $a \in \mathbb{R}^{n}$ with $|a|=R$. This contradicts (2.19), so (2.9) holds. If (2.15) fails then $\langle Q G(\bar{a}), J(\bar{a})\rangle>0$ for some $\bar{a} \in \partial U \cap X_{1}$ i.e. $|\bar{a}|_{0}=R$ and

$$
t\left\langle\int_{0}^{1} f(s, a) d s, a\right\rangle>0 \text { for all } t \in[0,1]
$$

Consequently $\left\langle\int_{0}^{1} f(s, a) d s, a\right\rangle>0$ for some $a \in \mathbb{R}^{n}$ with $|a|=R$. This contradicts 
(2.20), so (2.15) holds. In addition since $|u|_{0}<R$ for any solution $u$ to $(2.18)_{\lambda}$ we have that $(2.10)$ holds. Our result now follows from Theorem 2.4 with Remark 2.4.

Remark 2.5. Notice (2.20) can be replaced by any condition that will guarantee for us that $Q G \in M_{\partial U}(\bar{U}, E ; L, J P)$ is an essential map.

Remark 2.6. We let $M_{\partial U}^{*}(\bar{U}, E ; L, \Phi)$ denote the set of all continuous maps $F: \widetilde{U} \rightarrow E$ which satisfy the Mönch-Precup* condition (i.e. if $C \subseteq \bar{U}$ is countable, $W \subseteq k e r L$ is compact and $C \subseteq \overline{c o}\left(\{0\} \cup(L+\Phi)^{-1} F(C)\right)+W$ then $\bar{C}$ is compact) and with $(L-F)(x) \neq 0$ for $x \in \partial U \cap \operatorname{dom} L$. There are obvious analogues of Theorem 2.1, Theorem 2.3 and Theorem 2.4 in this case (we leave the details to the reader). It is of interest to note that " $(L+\Phi)^{-1} \Phi(\bar{U})$ is a bounded set in ker $L$ " is not needed in the analogue of Theorem 2.4 (however it is needed in the analogue of Theorem 2.3).

\section{§3. Set Valued Maps}

Let $X$ and $E$ be Fréchet spaces, $U$ an open subset of $X, 0 \in U$ and $\Phi \in H_{L}\left(X, E_{1}\right)$ is fixed (here $L$ and $E_{1}$ are as described in Section 1).

Definition 3.1. A multivalued map $F: \bar{U} \rightarrow 2^{E}$ (here $2^{E}$ denotes the family of nonempty subsets of $E)$ is said to be $(L, \Phi)$ upper semicontinuous if $(L+\Phi)^{-1} F: \bar{U} \rightarrow C K(X)$ is an upper semicontinuous (u.s.c.) map; here $C K(X)$ denotes the family of nonempty, compact, convex subsets of $X$.

Remark 3.1. It is possible to take $(L+\Phi)^{-1} F: \bar{U} \rightarrow A C(X)$ instead of $(L+\Phi)^{-1} F: \bar{U} \rightarrow C K(X)$ in the above definition and throughout this section (for example in Theorem 3.1); here $A C(X)$ denotes the family of nonempty, compact, acyclic subsets of $X$. Recall a subset $A$ of $X$ is acyclic if $H^{m}(A)=\delta_{0 m} \mathbb{Z}$, where $\left\{H^{m}\right\}_{m \in \mathbf{N}}$ denotes the Čech cohomology functor with integer coefficients.

Definition 3.2. A multivalued map $F: \bar{U} \rightarrow 2^{E}$ is said to be $(L, \Phi) k$-set contractive if $(L+\Phi)^{-1} F: \bar{U} \rightarrow C K(X)$ is a $k$-set contractive map.

Definition 3.3. We let $M M(\bar{U}, E ; L, \Phi)$ denote the set of $(L, \Phi)$ u.s.c., $k$-set contractive (here $0 \leq k<1$ ) maps $F: \bar{U} \rightarrow 2^{E}$ with $(L+\Phi)^{-1} F(\bar{U})$ a bounded set in $X$.

Remark 3.2. It is possible to discuss $(L, \Phi)$ condensing maps instead of $(L, \Phi) k$-set contractive maps in Definition 3.3 (and throughout this section). 
Remark 3.3. One could also discuss in this section (for example in Theorem 3.1) $(L, \Phi)$ admissible maps $[6],(L, \Phi)$ closed maps [7], and $(L, \Phi)$ approximable maps $[7,8]$.

Definition 3.4. We let $M M_{\partial U}(\bar{U}, E ; L, \Phi)$ denote the maps $F \in M M(\bar{U}, E ; L, \Phi)$ with $\operatorname{Lx\notin F}(x)$ for $x \in \partial U \cap \operatorname{dom} L$.

Definition 3.5. A map $F \in M M_{\partial v}(\bar{U}, E ; L, \Phi)$ is essential if for every $G \in M M_{\partial U}(\bar{U}, E ; L, \Phi)$ with $\left.G\right|_{\partial U}=\left.F\right|_{\partial U}$ we have that there exists $x \in \bar{U} \cap \operatorname{dom} L$ with $L x \in G(x)$.

Theorem 3.1. Let $X$ and $E$ be Fréchet spaces, $U$ an open subset of $X$, $0 \in U$ and $\Phi \in H_{L}\left(X, E_{1}\right)$. Suppose $F \in M M_{\partial v}(\bar{U}, E ; L, \Phi)$ is an essential map and $H: \bar{U} \times[0,1] \rightarrow C c(E)$ (here $C c(E)$ denotes the family of nonempty, closed subsets of $E)$ is a $(L, \Phi)$ u.s.c. map (i.e. $(L+\Phi)^{-1} H: \bar{U} \times[0,1] \rightarrow C c(E)$ is u.s.c) with the following properties:

$$
H(x, 0)=F(x) \quad \text { for } \quad x \in \bar{U}
$$

(3.2) $L x \notin H_{t}(x)$ for any $x \in \partial U \cap \operatorname{dom} L$ and $t \in(0,1]$ (here $\left.H_{t}(x)=H(x, t)\right)$

and

$$
\left\{\begin{array}{l}
\text { for any continuous } \mu: \bar{U} \rightarrow[0,1] \text { with } \mu(\partial U)=0 \text { the map } \\
\quad R_{\mu}: \bar{U} \rightarrow C c(E) \text { defined by } R_{\mu}(x)=H(x, \mu(x)) \text { is in } M M(\bar{U}, E ; L, \Phi) .
\end{array}\right.
$$

Then there exists $x \in U$ Udom $L$ with $L x \in H_{1}(x)$.

Proof. Let

$$
\begin{aligned}
B & =\left\{x \in \bar{U} \cap \operatorname{dom} L: L x \in H_{t}(x) \text { for some } t \in[0,1]\right\} \\
& =\left\{x \in \bar{U}: x \in(L+\Phi)^{-1}\left(H_{t}+\Phi\right)(x) \text { for some } t \in[0,1]\right\}
\end{aligned}
$$

As in Theorem 2.1, $B \neq \emptyset$. Moreover the continuity of $\Phi,(L+\Phi)^{-1}$ and the $(L, \Phi)$ upper semicontinuity of $H$ guarantees [1] that $B$ is closed. Then there exists a continuous $\mu: \bar{U} \rightarrow[0,1]$ with $\mu(\partial U)=0$ and $\mu(B)=1$. Define a map $R: \bar{U} \rightarrow C c(E)$ by

$$
R(x)=H(x, \mu(x))
$$

By (3.3) we have $R \in M M(\bar{U}, E ; L, \Phi)$. In addition for $x \in \partial U$ ndom $L$, 


$$
R(x)=H_{0}(x)=F(x)
$$

and so $R \in M M_{\partial U}(\bar{U}, E ; L, \Phi)$. Also since $\left.R\right|_{\partial U}=\left.H_{0}\right|_{\partial U}=\left.F\right|_{\partial U}$ and $F \in M M_{\partial U}$ $(\bar{U}, E ; L, \Phi)$ is essential there exists $x \in \bar{U} \cap \operatorname{dom} L$ with $L x \in R(x)$ (i.e. $L x \in H_{\mu(x)}(x)$ ). Thus $x \in B$ and so $\mu(x)=1$. Consequently $L x \in H_{1}(x)$.

Next we recall a result [3] from the literature.

Theorem 3.2. Let $X$ be a Fréchet space and let $D$ be a nonempty, closed, convex subset of $X$. Suppose $J_{0}: D \rightarrow C K(D)$ is a u.s.c., $k$-set contractive (here $0 \leq k<1)$ map with $J_{0}(D)$ a bounded set in $D$. Then $J_{0}$ has a fixed point in $D$.

Our next theorem extends a result of Volkmann [11 pp. 240].

Theorem 3.3. Let $X$ and $E$ be Fréchet spaces, $U$ an open subset of $X$, $0 \in U \cap \operatorname{dom} L$ and $\Phi \in H_{L}\left(X, E_{1}\right)$. Suppose $G: \bar{U} \rightarrow 2^{E}$ is a $(L, \Phi)$ u.s.c., $k$-set contractive (here $0 \leq k<1)$ map with $(L+\Phi)^{-1} G(\bar{U})$ a bounded set in ker $L$. In addition assume

$$
(L+\Phi)^{-1} \Phi(\bar{U}) \text { is a bounded set in } X
$$

and

$$
L x \notin t G(x)+(1-t)(-\Phi(x)) \text { for } x \in \partial U \cap \operatorname{dom} L \text { and } t \in(0,1)
$$

are satisfied. Then there exists $x \in \bar{U} \cap \operatorname{dom} L$ with $L x \in G(x)$.

Proof. We assume $L x \notin G(x)$ for $x \in \partial U \cap \operatorname{dom} L$ (otherwise we are finished). Then

$$
L x \notin t G(x)+(1-t)(-\Phi(x)) \text { for } x \in \partial U \cap \operatorname{dom} L \text { and } t \in[0,1] \text {. }
$$

Let $H(x, t)=t G(x)+(1-t)(-\Phi(x))$ for $(x, t) \in \bar{U} \times[0,1]$ and $F(x)=-\Phi(x)$ for $x \in \bar{U}$. It is clear that $H: \bar{U} \times[0,1] \rightarrow C c(E)$ is a $(L, \Phi)$ u.s.c. map. Also (3.1) and (3.2) hold. To see that (3.3) is true notice if $W \subseteq \bar{U}$ then

$$
(L+\Phi)^{-1} R_{\mu}(W) \subseteq \overline{c o}\left((L+\Phi)^{-1} G(W) \cup(L+\Phi)^{-1}(-\Phi(W))\right) .
$$

We now have immediately that $R_{\mu} \in M M(\bar{U}, E ; L, \Phi)$ and so (3.3) holds. We can apply Theorem 3.1 if we show $F \in M M_{\partial U}(\bar{U}, E ; L, \Phi)$ is essential. Notice it is immediate from (3.4) that $F \in M M_{\partial v}(\bar{U}, E ; L, \Phi)$ [Note if $L x_{0} \in F\left(x_{0}\right)$ for some $x_{0} \in \partial U \cap \operatorname{dom} L$ then $x_{0}=0$, a contradiction]. To show $F$ is essential let $\theta \in M M_{\partial U}(\bar{U}, E ; L, \Phi)$ with $\left.\theta\right|_{\partial U}=\left.F\right|_{\partial U}=-\left.\Phi\right|_{\partial U}$. Let $D=\overline{c o}\left((L+\Phi)^{-1}(\theta+\Phi)(\bar{U})\right)$ 
and let $J_{0}: D \rightarrow C K(D)$ be defined by

$$
J_{0}(x)=\left\{\begin{array}{l}
(L+\Phi)^{-1}(\theta+\Phi)(x), x \in \bar{U} \\
\{0\}, x \notin \bar{U} .
\end{array}\right.
$$

Note $0 \in D$ and it is easy to see that $J_{0}$ is a u.s.c., $k$-set contractive map (note $(L+\Phi)^{-1} \Phi(\Omega)$ is relatively compact for any bounded subset $\Omega$ of $\left.X\right)$ with $J_{0}(D)$ a bounded set in $D$. Theorem 3.2 implies that there exists $x \in D$ with $x \in J_{0}(x)$. Now if $x \notin U$, we have $x \in J_{0}(x)=\{0\}$, a contradiction. Thus $x \in U$ so $x \in J_{0}(x)=(L+\Phi)^{-1}(\theta+\Phi)(x)$ i.e. $x \in U$ तdom $L$ and $L x \in \theta(x)$. Hence $F$ is essential and we may apply Theorem 3.1 to deduce the result.

Remark 3.4. There is an obvious analogue of Theorem 2.4 in this setting. We leave the details to the reader. Also it is possible to apply our results to differential inclusions following the ideas in Section 2.

\section{References}

[ 1] Agarwal, R. P. and O'Regan, D., Homotopy and existence of solutions for the nonlinear equation $L x \in N x$, to appear in Nonlinear Anal.

[2] Deimling, K., Nonlinear functional analysis, Springer Verlag, New York, 1985.

[3] Fitzpatrick, P. M. and Petryshyn, W. N., Fixed point theorems for multivalued noncompact a cyclic mappings, Pacific Jour. Math., 54 (1974), 17-23.

[4] Granas, A., Sur la méthode de continuité de Poincare, C. R. Acad. Sci. Paris, 282 (1976), 983-985.

[5] Mönch, H., Boundary value problems for nonlinear ordinary differential equations of second order in Banach spaces, Nonlinear Anal., 4 (1980), 985-999.

[6] O'Regan, D., Coincidences for admissible and $\Phi^{*}$ maps and minimax inequalities, J. Math. Anal. Appl., 220 (1998), 322-333.

[7] — Fixed point theory for closed multifunctions, Arch. Math., 34 (1998), 191-197.

[8] - Fixed points and random fixed points for weakly inward approximable maps, Proc. Amer. Math. Soc., 126 (1998), 3045-3053.

[9] Precup, R., On the topological transversality principle, Nonlinear Anal., 20 (1993), 1-9.

[10] Treves, F., Topological vector spaces, distributions and kernels, Academic Press, New York, 1967.

[11] Volkmann, P., Démonstration d'un théoreme de coincidence par la méthode de Granas, Bull. Soc. Math. Belgique Ser. B, 36 (1984), 235-242. 